\title{
Médicos, administradores y curanderos. Tensiones y conflictos al interior del arte de curar diplomado en la provincia de Santa Fe, Argentina (1861-1902)/
}

\author{
Doctors, Administrators and Curanderos. \\ Tensions and Conflicts among the Oficial Healing Art \\ in the Province of Santa Fe, Argentina (1861-1902)
}

José Ignacio Allevi ORCID iD: http://orcid.org/0000-0003-4819-1398

CONICET / ISHIR-CESOR / UNR, Argentina

Adrián Carlos Alfredo Carbonetti ORCID iD: http://orcid.org/0000-0002-2093-2046

CONICET / CIECS / UNC, Argentina

Paula María Sedrán ORCID iD: http://orcid.org/0000-0002-0592-0397

CONICET / ISHIR-CESOR, Argentina

El trabajo analiza una serie de recursos administrativos presentados ante las autoridades de la provincia de Santa Fe bien por los médicos diplomados para denunciar el ejercicio ilegal de la medicina — curanderismo- - bien por los vecinos para solicitar se autoricen este tipo de prácticas no permitidas. Nos interesa tensionar el proceso de medicalización social mostrando la pervivencia y legitimidad de ciertas prácticas sustentadas en saberes "populares», así como la incertidumbre de los saberes biomédicos. Por otra parte, discutiremos el proceso de profesionalización de los médicos como expertos «únicos» en la prestación de servicios de salud, al mostrar que las prácticas «alternativas» de curar eran en verdad la norma de los ciudadanos.

Palabras Clave: Curanderismo; Profesionalización; Medicalización; Argentina.

The paper analyzes a series of administrative appeals presented to the authorities of the province of Santa Fe either by qualified doctors to denounce the illegal exercise of medicine — known as curanderismo- as well as by neighbors to request authorization of this type of unallowed practices. We are interested in stressing the process of social medicalization by showing the survival and legitimacy of certain practices based on "popular» knowledges, and the uncertainty of biomedical knowledge. On the other hand, we will discuss the professionalization process of physicians as «unique» experts in the provision of health services, showing that «alternative» healing practices were indeed the norm of citizens.

Keywords: Curanderismo; Profesionalization; Medicalization; Argentina.

Copyright: (C) 2018 CSIC. Este es un artículo de acceso abierto distribuido bajo los términos de la licencia de uso y distribución Creative Commons Reconocimiento 4.0 Internacional (CC BY 4.0). 


\section{La profesión como punto de llegada: derivas de la legitimidad médica}

Este trabajo se propone constituir un aporte a las discusiones que renuevan constantemente el campo de la historia social y cultural de la salud y la enfermedad, al discutir la complejidad de dos procesos: la medicalización social, por una parte, y la profesionalización y legitimidad médica, por otra. ${ }^{1}$ De manera secundaria, entendemos que el problema de la legitimación y profesionalización de la medicina como saber hegemónico sobre la salud a partir de la tensión entre los diplomados y los sanadores empíricos puede aportar a otro campo de estudios. Nos referimos a la revisión historiográfica en torno al Estado como figura y actor omnicomprensivo, el cual ha sido revisitado y renovado en los últimos años en pesquisas que han enfatizado no solo la fragilidad de su tendido y la cuestión central de sus cuadros burocráticos, ${ }^{2}$ sino también el problema de los límites de la medicalización, en función de la efectividad de sus estructuras en el control y gestión de poblaciones tanto en zonas caracterizadas por la escasez de personal o de agencias específicas - problema estudiado para los Territorios Nacionales en Argentina-,${ }^{3}$ como también en las ciudades «centrales». En este sentido, nuestra propuesta responde a este tipo de inquietudes, en tanto la problemática del curanderismo se inserta en una miríada de planos desde los cuales observar cuestiones atinentes a la conformación Estado, a las modernizaciones sociales y al proceso de medicalización de distintas esferas de lo social.

La segunda mitad del siglo XIX en Argentina acarreó una serie de transformaciones que modificaron completamente el amplio espectro de la relación social: sociedad, Estado, economía y cultura, todos ellos atravesaron cambios sustanciales, a partir de lo que se entendió como procesos de modernización. ${ }^{4}$ Estos, sin embargo, no resultaron inocuos a todos los sectores sociales, y sus consecuencias derivaron en la emergencia de lo que numerosos estudios han entendido como «cuestión social». ${ }^{5}$

En este marco, los brotes epidémicos que azotaron a la población ingresaron en la agenda de la limitada acción del Estado, y con ello los médicos comenzaron a demandar un reconocimiento de su labor y su saber por este último. Como parte de este proceso de múltiples cambios, los médicos diplomados —inmigrantes, en su mayoría - fueron emergiendo

1 Carbonetti, Aizenberg y Rodríguez, 2014. Di Liscia, 2008. Armus, 2002.

2 Bohoslavsky y Soprano, 2010. Bohoslavsky y Godoy, 2010. Plotkin y Zimmermann, 2012.

3 Bohoslavsky y Di Liscia, 2008.

4 Bonaudo, 2007. Reguera, 2009.

5 Castel, 1997. Suriano, 2000. 
tímidamente como un actor que pretendía hegemonizar los sentidos y prácticas del arte de curar, frente a un número no menor de sujetos que tradicionalmente se habían abocado a estas tareas a partir de saberes empíricos y que contaban, además, con una legitimidad social considerable. ${ }^{6}$

Este grupo de médicos diplomados en vías de profesionalización era, a la sazón, parte de la elite local en los distintos espacios donde se encontraban, lo cual habilitó su posibilidad de ingresar en los espacios que en los distintos niveles del Estado fueron emergiendo para velar tanto por la salud de la población como por sus intereses corporativos — controlando títulos, habilitando farmacias y establecimientos de salud, etc.—. Los más relevantes en este sentido fueron el Departamento Nacional de Higiene y los Consejos de Higiene provinciales, instituciones nacidas al calor de crisis epidémicas o sanitarias que pugnaban por atender a una de las aristas de la cuestión social desde la higiene, así como también a resguardar el campo de acción de los diplomados e intentar construir progresivamente su monopolio. ${ }^{7}$ No obstante la ventaja relativa que suponía su vínculo con actores clave del Estado, como todo proceso, la profesionalización de la medicina fue un camino sinuoso y de múltiples vías, estudiado principalmente para el caso de Buenos Aires. ${ }^{8}$

Las dificultades de los diplomados en este sentido no eran menores, y si bien, por una parte, respondían a las limitaciones propiamente estatales en la capacidad - y voluntad - de control por sus instituciones y agentes, por otro lado no debemos perder de vista que tanto las intervenciones de la medicina académica o de las terapias populares-tradicionales eran inciertas en sus fundamentos y resultados, y así partir del hecho que la legitimidad de las prácticas médicas no superaba a la de curanderos, sanadores, hipnotistas, espiritistas, parteras, entre otros. Los estudios sobre la materia destacan que en el proceso de hegemonización médica fueron centrales las políticas de «des-calificación» y represión de prácticas que aparecían como «heterogéneas» frente a una supuesta homogeneidad y fortaleza del saber médico diplomado. ${ }^{9}$ En la disputa por la significación que los médicos debían librar para alcanzar no solo el monopolio de una práctica sino la legitimidad social de su saber, reconocemos dos aspectos analíticos: la definición simbólica de qué prácticas eran «efectivas» y, por otro lado, de cuáles serían «permitidas».

6 Di Liscia, 2003.

7 Prieto, 1996. Pascual, 2017. 2003.

8 González Leandri, 1999; 2012. Souza, 2008. Para el caso de Córdoba, véase Rodríguez,

9 Bubello, 2010. 
Estos estudios se interrogan sobre objetos diversos, desde el grado de efectividad que el ejercicio de la autoridad del Estado tuvo con respecto a las prácticas de medicina no diplomada, popular o curanderismo, hasta la específica forma en que la aparición de los Consejos de Higiene ligada a coyunturas sanitarias y políticas ${ }^{10}$ y la construcción de los curanderos como sus primeros enemigos indica el lugar que la salud pública ocupó en los ciclos de atención del Estado en formación. ${ }^{11}$ Estos interrogantes están vinculados a los procesos de conformación de la profesión médica dotada de legitimidad académica, pero principalmente social a escala local y regional. Así, los médicos «oficiales» fueron logrando que sus propuestas ganaran peso en las políticas públicas, ${ }^{12}$ posición desde la cual abogaron por el cumplimiento de las condiciones que la medicina académica debía reunir. Lo antedicho pone de manifiesto que los estudios existentes sobre curanderos, sanadores y la medicina popular no arrojan — aun — una visión general del tema, como así tampoco de la problemática específica de la tensión entre estos y los médicos. Este trabajo dialoga con dichos aportes, y pretende analizar una de las aristas de la profesionalización médica y la medicalización social, como fueron los numerosos intentos de reprimir y limitar el accionar de sanadores tradicionales.

La conflictividad que signó la relación entre médicos y curanderos se ha constituido en objeto de indagación histórica en los últimos años, ${ }^{13}$ en estudios que - a pesar del carácter fragmentario que presentan - han superado ciertos enfoques que pensaban el «triunfo» de la medicina diplomada atribuyendo un carácter a la vez performativo y efectivo a la letra de las normas que regulaban la praxis médica desde el siglo XVIII. ${ }^{14} \mathrm{Al}-$ gunos casos, por otra parte, resultan ejemplares de una serie de procesos subyacentes a la figuración pública de estos personajes ya avanzado el siglo XX, como el del profesor Carbell de Costa Rica en la década de $1930 .^{15}$

10 González Leandri, 2004. Pascual, 2017. Prieto, 1997.

11 Rodríguez, Carbonetti y Andreatta, 2013.

12 Carbonetti, 1997. González Leandri, 2006.

13 Sowell, 2002. Ferreira, 2003. Armus, 2016.

14 Amodio, 1997.

15 En efecto, Carbell, reunía una serie de saberes que vinculaban el plano espiritual y el médico, y al momento de ser denunciado por la policía de Salubridad presentó su caso no solo con un número importante de testimonios a su favor, sino que realizó un uso estratégico de una miríada de medios escritos (Molina y Palmer, 1996). En su análisis, Palmer muestra que el éxito de Carbell y su persecución por los médicos diplomados era en realidad el espejo de un proceso sobre el eclecticismo médico que se presentaba como una nueva dimensión de la medicina popular, al tiempo que le permitía dar cuenta de un particular proceso de imbricación de los mundos privados y públicos y la política, a la luz del fenómeno que el autor abordó como «populismo médico» (Palmer, 2002). 
La historiografía argentina ha relevado algunos casos significativos, donde el análisis de los sujetos que curaban fuera de la tradición académica se enfocó en el curanderismo o el parterismo, como son los estudios sobre casos de las actuales provincias de Tucumán, ${ }^{16} \mathrm{Jujuy},{ }^{17}$ Córdoba, ${ }^{18}$ Territorios Nacionales ${ }^{19}$ y Buenos Aires. ${ }^{20}$ Una figura vinculada a estos trayectos es la de los «charlatanes» en la cultura científica decimonónica, abordada prolíficamente en una miríada de estudios que se han preocupado por señalar su presencia, destacar las dificultades documentales de su rastreo, y fundamentalmente enfatizar el carácter trasnacionalizado e itinerante de los saberes que componen la ciencia «oficial», dando cuenta así de la mutua imbricación existente entre conocimientos del mundo popular y su recepción y resignificación en el campo de la medicina erudita. ${ }^{21}$ Sin embargo en este artículo no profundizaremos esta rica perspectiva analítica sino que buscaremos analizar los conflictos que se suscitaban entre médicos académicos (que podían ejercer gracias a la reválida de los órganos estatales) y falsos médicos o curanderos.

Va de suyo que el objeto de estos trabajos afronta dificultades documentales, que en buena medida han marcado el ritmo de constitución del sub campo historiográfico de la salud y la enfermedad. Para el caso del tenso vínculo entre una medicina tímidamente oficial y otra de carácter empírico y «popular», las investigaciones comparten limitaciones con el conjunto de los estudios sociales del siglo XIX y pueden pensarse en un doble registro: por una parte, escasez o discontinuidad documental; por otra, el hecho de que la gran mayoría de dichos documentos fueron producidos por elites «estatales». En este último punto, sin embargo, veremos que el ejercicio del derecho de petición a las autoridades por parte de los vecinos no fue una práctica menor, ${ }^{22}$ así como también que las propias fuentes «oficiales» dan cuenta de ciertas lagunas y de situaciones donde el marco legal y administrativo era puesto en segundo plano por parte de los cuadros estatales.

Por otra parte, es preciso señalar que los trabajos existentes reúnen dos condiciones que dan cuenta de su relevancia historiográfica. En primer término, y en tensión con las miradas generales, ponen en relieve los

16 Fabermann, 2005.

17 Fleitas, 2007.

18 Carbonetti, 1997.

19 Di Liscia, 2003.

20 Armus, 2007. Dahhur, 2013.

21 Podgorny, 2012; 2015.

22 Garcilazo, 2014. 
rasgos propios de las regiones estudiadas, un planteamiento que responde a un debate más que saldado en la pesquisa histórica contemporánea que ha revisitado el carácter territorial del enfoque regional para pensarlo como perspectiva teórico-metodológica de trabajo ${ }^{23}$ En segundo lugar, metodológicamente rescatan el estudio del «acontecimiento» como parte constitutiva de la trama social, permitiendo el trazado de una cartografía de lo social bajo una clave más densa. ${ }^{24}$ Este trabajo busca hacerse eco de estas visiones para analizar un fenómeno general bajo la lógica de instancias particulares y discontinuas de lo social en el espacio abordado.

El conjunto de casos empíricos que analizamos en este artículo transcurren en el territorio de la actual provincia de Santa Fe, Argentina, durante la segunda mitad del siglo XIX. ${ }^{25}$ Cabe señalar, en este sentido, que el corpus de fuentes utilizado fue reunido a partir de expedientes administrativos, judiciales y civiles, lo cual nos permite iluminar aspectos del proceso de medicalización en esferas del plano cotidiano entre tantos otros posibles. Una de las mayores potencialidades de los archivos en este período de indefiniciones de la autoridad estatal reside en la cotidianidad que permiten inferir sobre los sujetos involucrados, así como su capacidad de crítica sobre los documentos oficiales elaborados desde las elites y las agencias que ocupaban. ${ }^{26}$

En un contexto de integración del país al sistema capitalista y de sus particularidades geo-climáticas, la provincia de Santa Fe fue una pieza clave en el proyecto productor de materias primas que definió su incorporación. Esto no implicó, empero, un proceso homogéneo para el conjunto del territorio provincial, donde se distinguen tres regiones de ocupación y explotación del suelo, con el arribo de flujos migratorios externos e internos y con las transformaciones sociales consecuentes: el sur pampeano, con una fuerte impronta de grandes propietarios absentistas que encontraron en los negocios inmobiliarios rurales y en el arrendamiento pingües ganancias; la franja transicional central, con el predominio de pequeñas y medianas propiedades abocadas a la producción agrícola o ganadera intensivas; y el

23 Fernández y Dalla Corte, 2001. Fernández, 2007; 2008.

24 Revel, 2005 .

25 Hemos procurado realizar una transcripción literal de las fuentes abordadas, en tanto entendemos que reflejan cambios significativos en el uso de la lengua. Dada la cantidad de diferencias que presenta la escritura y ortografía con el sistema actual, hemos obviado agregar la aclaración luego de cada una de ellas.

26 Otros trabajos han abordado el problema del curanderismo desde fuentes diversas, como la prensa, véase Rivero, Carbonetti y Rodríguez, 2017. 
norte chaqueño, con escaso poblamiento, abundancia de recursos boscosos y pobre fertilidad de los suelos, lo cual orientó su economía hacia la actividad forestal y de extracción de tanino. ${ }^{27}$

\section{El control de las prácticas de curar: un camino espinoso}

A partir de la segunda mitad del siglo XIX, momento en que los actuales territorios provinciales comenzaron a ganar estabilidad administrativa luego de los conflictos post independencia que atravesaron al antiguo virreinato del Río de La Plata, encontramos algunos registros del incipiente problema de la legitimidad de quienes ejercían prácticas de curar. Podemos diferenciar, en este sentido, un primer panorama hacia fines de la sexta década del siglo. En 1861, Teófilo Romang, médico diplomado, fue «recibido» por el Concejo Municipal de la colonia agrícola de Esperanza como médico del pueblo, asignándole el pago de cuatrocientos pesos bolivianos anuales. Como dicha suma nunca le fue abonada por falta de recursos del municipio, el galeno comenzó a cobrar honorarios particulares a los ciudadanos por sus prestaciones. Tres años después de su llegada, empero, vemos el primer registro documentado de la conflictividad entre los médicos diplomados del territorio provincial y los practicantes del arte de curar no académico cuando Romang se dirige al gobernador para denunciar a Felipe Vehr por ejercicio ilegal de la medicina:

Pero hace ya tiempo que el colono Felipe Vehr se ha permitido sin autorización ninguna a curar enfermos y componer algunos miembros quebrados, cuyos he tenido a varias veces que quebrar en segundo para poder componerlos, y también se ha permitido hablar mal de mi persona desacreditándome a los demás colonos, particularmente de mi oficio y arte. ${ }^{28}$

Aquí se juegan dos factores en la denuncia de Romang. Por una parte, el simple hecho de que, ante el cobro por sus prácticas, es más que probable que Vehr no solo haya tenido una presencia más arraigada en el lugar sino que además haya solicitado un pago más modesto, presumiblemente a partir del trueque, como veremos ocurría con otros curanderos. Otro tanto se dirimía desde el plano de la reputación de Romang, quien de esa manera lo

27 Hourcade y Godoy, 1993. Gallo, 2004.

28 Archivo General de la Provincia de Santa Fe (en adelante AGPSF), Ministerio de Gobierno (MG), tomo 25, 1864, exp. 16. 
expresa en su solicitud : «Por esos motivos vengo a solicitar de V.E. dignase ordenar por medio del Sr. Juez de Paz a dicho Felipe Vehr que cesa tales hechos sin que me veré en la obligación de dejar la colonia a la merced de un charlatán según lo dice el Sr. Juez de Paz en su declaración...». ${ }^{29}$

En el mismo año, los vecinos de Coronda enfrentan un cuadro epidémico a partir del cual apelan al gobernador solicitando auxilio. Observemos aquí no solo la escasa disponibilidad de profesionales para actuar en contextos críticos, sino también las concepciones sobre la enfermedad plasmadas en la solicitud:

hace algún tiempo a que este vecindario sufre el flagelo de enfermedades que por su desarrollo estraordinario en el vecindario se deja conocer ser ya de carácter epidémico sin que se haya notado disminución alguna, sin embargo del cambio de estación y de haber llovido algunas veces; motivos suficientes que podrían haber contribuido a su total desaparición pues, que sin embargo de la templanza de la admósfera parece que hoy se presenta de un modo recrudecido manifestándose sucesibamente bajo nuevos síntomas que tienen alarmada a la población. ${ }^{30}$

Ante este cuadro, los diecinueve vecinos que firman la petición solicitan un médico más que colabore con el que ya se encontraba en el poblado:

En tal conflicto ocurrimos a la paternal protección de V.E. para que atendida nuestra demanda, se digne destinarnos un facultativo de Medecina ya sea de los que existen en la Capital o de los del Rosario, en la forma y manera que lo estime conveniente, al fin de que asociado al ciudadano Dn Felipe Fernandez el único con el que actualmente cuenta la población como médico, puedan en cuanto les sea posible contener el desarrollo de dichos males, o buscar algún genero de preservación ${ }^{31}$

Apenas unos años más tarde, en 1867, desde el Departamento de San José y Calchines se dirigen al gobernador apelando a una solución más práctica que el envío de un diplomado: precisamente ante la falta de médico y la presencia de un curandero, solicitan que este último sea habilitado para poder ejercer:

que caresiendo en los referidos Departamentos, de un médico, para atender a los enfermos, pues lo exije ya el cresido número de habitantes, que no vajaron de dos mil quinientos á tres mil, venimos ante V.E. a pedir al Sr. Dn. Visente Bital por ser ya conosido de todos, y haber echo acá varias curas notables, lo que nos prueba los buenos conosimientos prácticos que tiene, y á mas de esto, lo umanitario y desinteresado que

29 Idem.

30 Idem.

31 Idem. 
se á manifestado, por todo lo espuesto esperamos que V.E. accederá a nuestra solicitud, por la escasez que tenemos de médico, y por que en este tenemos confianza. Por tanto a V.E. suplicamos se digne a concedernos esta gracia. ${ }^{32}$

Estos casos presentan un panorama donde es posible observar las primeras iniciativas de la medicina diplomada por disputar un lugar legítimo a los practicantes populares, que, como resulta evidente, contaban con un nivel de inserción comunal y hasta con mayor autoridad que los incipientes practicantes «oficiales». Junto al problema de la legitimidad emergía un segundo aspecto, la escasez de médicos letrados para «competir» con los curanderos, así como los límites concretos del «brazo» del Estado a la hora de asegurar un lugar para estos últimos.

Los reclamos tanto de los vecinos como de los actores médicos, por otro lado, tuvieron eco recién hacia 1868, año bisagra para la profesión en la provincia, al menos en términos institucionales. Motorizados a partir de la epidemia de cólera del año anterior, en dicho año se crearon los Consejos de Higiene de Santa Fe y Rosario, así como la Asistencia Pública de esta última ciudad. ${ }^{33}$ Concebidos para monopolizar la atención de la salud desde el Estado, así como el control de las prácticas de los particulares, entre sus objetivos señalaban el control de títulos y autorización del ejercicio de los ramos menores - como las parteras o flebótomos - y de las farmacias, la visita de establecimientos en los contextos de brotes epidémicos y fundamentalmente el «celar» el ejercicio profesional de los galenos. Considerando la escasez de practicantes diplomados, fue corriente la inserción y autorización de médicos extranjeros, a los que más adelante se les solicitaba un examen de reválida. ${ }^{34}$

Casi veinte años después veremos un funcionamiento distinto en la lógica de las denuncias por parte de los galenos, que si bien contaban ya con un cierto respaldo institucional, su legitimidad aun no lograba decantar en el tejido social. Vemos así que, nuevamente en la localidad de Esperanza, el primero de abril de 1886 un importante número de médicos y farmacéuticos se dirigieron a las autoridades denunciando las dimensiones y el accionar de los curanderos en la zona. De acuerdo con la descripción que realizan, el problema tendría una extensión considerable en tanto afectaba a mayores actores, puesto que no solo realizaban prácticas curativas, sino que además

32 AGPSF, MG, tomo 31, 1867.

33 Prieto, 1996.

34 Registro Oficial Santa Fe, tomo VI, 1867-1869, 240-245. 
sugerirían el consumo de ciertos medicamentos no reconocidos por el Consejo de Higiene —y por ende «ilegales»—, los cuales serían expedidos en comercios que tampoco estaban autorizados por la institución:

\begin{abstract}
Al Sr. Gefe Político del Departamento Las Colonias [...] Los abajo firmantes, Doctores en Medicina y Cirugia, y farmacéutico establecidos en este pueblo, y habilitados legalmente para el ejercicio de nuestra profesión por autorización del tribunal de $\mathrm{Me}$ dicina de la Capital, ante V.E, con el debido respeto nos presentamos y esponemos que: Habiendo tomado el curanderismo grandes proporciones con gran detrimento de la Salud de este pueblo, llegando el atrevimiento de algunos curanderos hasta el punto de usar medicamentos heroicos con daños crecidísimos, y esponer de este modo la vida de los enfermos y existiendo a mas establecimientos que bajo el nombre de Farmacias o Boticas, espenden estos medicamentos peligrosos sin estar debidamente autorizados; y siendo estrictamente prohibido por la Ley, el ejercicio de Medicina o Farmacia en la Provincia a personas no autorizadas, rogamos a V.E. por salvaguardia de la población, y defensa de nuestros derechos legítimamente adquiridos, se sirva hacer citar a las personas que hoy dícense ocupar indebidamente en el ejercicio de la Medicina y Farmacia, y les ordene que inmediatamente obtengan la autorización correspondiente del Tribunal de Medicina de Santa Fe, o cesar en el ejercicio de un ramo que no les corresponde, bajo las formas que la Ley establece. ${ }^{35}$
\end{abstract}

Como podemos ver, ha cambiado la lógica de la argumentación. Por una parte, llama la atención la apelación a un derecho obtenido ya en términos corporativos, donde la actividad profesional se incluye con todos sus matices - práctica médica y farmacia-. Un segundo aspecto tiene que ver con la apelación al riesgo de dichos servicios y productos sobre la población como motivaciones de su requerimiento. Por último, y no menos importante, esta denuncia revela el hecho de que, junto con ciertas prestaciones no oficiales que era preciso detener para resguardar el saber y el campo laboral de los especialistas, se abría la necesidad de regular y resguardar el consumo de aquellas sustancias que la medicina delimitaba como efectivas en términos terapéuticos. Esto es, no solo el acceso a la «salud» vendría de la mano del médico autorizado, sino también el consumo de aquellas sustancias que la cultura «popular» local utilizaba como apoyos al proceso curativo.

Ante la solicitud de los miembros locales de la corporación, el jefe político solicitó asesoramiento al gobierno de la provincia, donde le indicaron «convocar a un despacho a todos los médicos (o que pasen por tales) y Farmacéuticos y exigirles un correspondiente diploma o permiso del tribunal

35 AGPSF, MG, tomo 88, 1887, exp. 22. 
de medicina de esta capital. Los que no lo exhibieren deben sufrir la multa legal y su respectivo apercibimiento». Ahora bien, al tomar conocimiento de la cuestión, el Consejo de Higiene interpuso su visión del asunto, que expresaba, por una parte, su voluntad de ganar mayor peso como instancia regulatoria y, por otra, su voluntad de contar con la colaboración tanto de funcionarios políticos como de los cuadros de seguridad para ejercer el control de las prácticas de curar. Este último punto será, como veremos, uno de los mayores obstáculos con que contarían, dada la inscripción local de los colaboradores requeridos.

Sr. Ministro: Siendo justísima la solicitud de los Médicos y Farmacéuticos de la Esperanza, el Consejo de Higiene cree que debe pasarse no al Gefe Político de esa ciudad para que ponga en conocimiento de los que ejercen la profesión de Médicos o Farmacéuticos, sin ser recibidos en alguna de las Facultades de la república que se presenten a este Consejo entes del término de un mes renovar la autorización si la tienen o solicitarla para el futuro. Los abusos cometidos por curanderos y boticarios son sin cuenta, por lo que si V.E., como este Consejo, considera de necesidad cortar de raíz los medios que a ciertas personas sirven para explotar la ignorancia del pueblo y a fin de garantir en lo posible la salud del pobre facilmente engañado, convendría oficiar a las autoridades de los Departamentos San José, San Javier, Las Colonias y San Gerónimo que corresponden a la jurisdicción del Consejo de Higiene de la Capital en igual sentido que al de la Colonia Esperanza, así como que no concedan patente de médico y Farmacéutico a los que no presenten diploma o autorización de este Consejo; de esta manera estado prevenidos pueden hacerse efectivas las multas. Es cuanto tiene que informar este consejo de Higiene al Sr. Ministro. Candido Pujato. ${ }^{36}$

Esta actitud por parte del presidente del Consejo pone en evidencia algunas cuestiones. Si bien por una parte traduce una serie de cambios que habían cristalizado en la nueva estructura que adoptó el Consejo de Higiene a partir de su reforma en 1887, por otra parte da cuenta de la competencia que estos sanadores empíricos representaban para las prácticas «oficiales», y de allí la necesidad de contener y reprimir dichas prácticas. En términos de su estructura, el Consejo centralizó sus actividades en la capital provincial, subordinando la sede existente en la ciudad de Rosario a este último. Además, luego de casi dos décadas de funcionamiento, el organismo redefinía sus funciones respecto a la corporación médica así como su posición en y para el Estado. Si, por un lado, reforzaba su potestad de autorización de títulos extranjeros, el avalúo de honorarios médicos, su lugar en la aplicación de penas por ejercicios irregulares — donde se incluía la mediación

36 Idem. 
y legitimación de las mismas por parte de un abogado y un juez del crimen-, o su control sobre las condiciones edilicias higiénicas en farmacias y la puesta en uso de la farmacopea francesa, por otro lado hay indicios que muestran su orientación como una agencia y un saber orientados hacia el Estado. Además de que sus miembros ahora eran designados por el poder ejecutivo y tanto estos como los inspectores percibían una remuneración, entre sus nuevos objetivos el Consejo se propone elaborar una estadística médica, brindar asesoramiento a municipios y comisiones de fomento, la posibilidad de nombrar comisiones higiénicas cuando sean requeridas, la imposición a los diplomados de informar cualquier indicio de brote epidémico y un refuerzo sobre sus actividades de inspección de establecimientos médicos y especialmente farmacéuticos. ${ }^{37}$

\section{¿Curanderos o médicos ilegales? Disputas sobre el monopolio de curar}

Estos cambios marcan una nueva actitud respecto al control y persecución de las prácticas «ilegales» de ejercicio de la medicina, y el caso que inaugura este período del Consejo de Higiene lleva una doble particularidad. Por un lado, por el tenor de la denuncia - llevada a los límites del paroxismo-; por otro, por ser el primer caso donde, ante las sucesivas misivas y multas aplicadas por el Consejo, el curandero acusado responde con una denuncia contra su presidente, en la cual un asesor letrado expuso una serie de argumentos legales para legitimar su derecho a ejercer una práctica. ${ }^{38}$ Se trata de Juan Pablo Quinteros, quien no era curandero sino espiritista, y cuyo caso ha sido objeto de otros trabajos. ${ }^{39}$

Años más tarde, el 9 de octubre de 1894 Guillermo Ovalle, médico de policía de Helvecia — localidad del norte provincial—, se dirige al ministro de Gobierno provincial para denunciar ciertas irregularidades que no atañen ya solo a la prestación de servicios por parte de los «sanadores» locales, sino también a la necesidad de controlar ciertas prácticas que se buscaba secularizar y ubicar bajo la órbita del Estado, como el registro de defunciones:

37 Registro Oficial Santa Fe, 1887, 159-167.

38 «Atanacio Páez en representación de Juan P. Quinteros contra el Consejo de Higiene», AGPSF, MG, tomo 115, 1887, exp. 30. «Vecinos solicitan se autorice a Juan P. Quinteros», AGPSF, MG, tomo 88, 1887, exp. 22, n. ${ }^{\circ} 6489$.

39 Sedrán, Carbonetti y Allevi, en prensa. 
Al objeto de evitar aquellos y poner trabas á un sin número de curanderos que egercen la medicina sin titulo ni permiso alguno, solicito de SS. disponga (salvo su mejor parecer) que esta Comision de Fomentos no consienta en el enterramiento de ningun cadáver sin certificación del reconocimiento facultativo, pudiendo saberse asi las enfermedades que predominan en el pueblo, el caracter que revistan y, en casos epidémicos tomar el Médico de Policia que suscribe de acuerdo con el H. Consejo de Higiene todas aquellas medidas que la Ciencia y la Higiene establecen. Por otra parte, y siguiendo marcha tan anómala como hasta hoy se sigue pueden cometerse crímenes dejando vurlada la acción judicial; en su virtud y atendiendo a lo que dejo consignado, vengo a pedir a SS. lo solisitado si lo cree en justicia... ${ }^{40}$

Apenas unos meses después, se desató una misiva interesante en la localidad de Sunchales por el contexto donde se desplegó así como por el accionar del Consejo, donde Manuel Martínez Olano fue acusado por ejercicio ilegal de la medicina. El 12 de diciembre de 1894 el presidente del organismo solicitó al juez de paz de la localidad que gestionase el cobro de una multa al sujeto en cuestión por ejercer la medicina de forma ilegal, habiendo sido apercibido previamente:

Comprobándose nuevamente que el Sr. Martinez Olano ejerce la medicina, no obstante prohibicion anterior, se ha resuelto aplicarle una multa de doscisntos pesos moneda nac., cantidad que hará usted efectiva trabando embargo de sus bienes en caso se niegue a satisfacerla. El resultado de sus diligencias se servirá hacermelo conocer por primer correo. ${ }^{41}$

Dos días después, el magistrado informa sobre la recepción de la solicitud y del procedimiento efectuado. Es importante que retengamos el devenir de los acontecimientos aquí. Cuando informan a Martínez Olano de la multa, este alega no tener medios para abonarla, lo cual es ratificado por dos testigos, y con ello se da por finalizado el asunto:

En la misma fecha me constituí al domicilio del Sr. Martinez Olano, a quien notifique de la precedente resolución del honorable Consejo, intimándole el pago de la multa establecida, y habiéndose negado a pagarla, le requerí bienes a embargo; a lo cual tambien contestó que no tenía ningunos. Resultando de las diligencias practicadas que no posee otros bienes que los muebles de su preciso uso, se dio por terminado este acto que lo firmó el notificado y los testigos suscritos por ante mi de quien certifico. ${ }^{42}$

40 «Guillermo Ovalle se presenta en queja contra los curanderos de Helvecia», AGPSF, MG, tomo 320,1894 , exp. 42.

41 AGPSF, MG, tomo 30, 1895, exp. 30

42 Idem. 
El resultado de la requisitoria, firmada por Martínez Olano, dos testigos y el juez de paz involucrado, no satisfizo al presidente del Consejo, quien apenas unos días después recurrió a la autoridad jerárquica superior al juez de paz, esto es, el jefe político del departamento de Rafaela, para que comprobara si las diligencias fueron certeras o no:

Por segunda vez el Consejo se sirve solicitar su concurso para hacer efectivas las medidas tomadas contra del curandero Sr. Manuel Martínez Olano. Hoy se trata de saber si las diligencias hechas por el Juez de Paz de Sunchalez son exactas. Sírvase ordenar las averiguaciones necesarias, y si resultaran falsas, disponer se efectue la multa de \$200.- por los medios que el Jefe Político estime más rápidos y seguros. Saludalo atentamente. Candido Pujato. ${ }^{43}$

En medio de esta nueva solicitud de Pujato, acontece algo que evidencia la incongruencia entre la pretendida legitimidad que este reclamaba para la corporación médica y la legitimidad y peso local de las figuras asociadas a la prestación de servicios de salud en las comunidades. El 30 de diciembre de 1894, el comisario general del poblado se dirige en un telegrama al ministro de Gobierno - por encima del Consejo de Higiene - notificando sobre un brote epidémico de cólera ante el cual, por falta de médico, propone se habilite a este sujeto que ya contaba con la negativa del órgano de la corporación:

Sr. Ministro de Gobierno de S. Fe. Oficial. Participo a SS. que esta población está alarmada con la epidemia del cólera y la falta de médico por haberse ausentado el que había aquí esta el Dr Manuel Martinez Olano pero como consejo de higiene ha ordenado que no cure. Digame si SS. le podrá permitir en vista de las circunstancias por que atraviesa esta población. Saludo a SS. ${ }^{44}$

La tensión que las medidas del Consejo despertaban al evidenciar su fragilidad institucional y la escasa cobertura que alcanzaba en contextos normales se agudizaba en tiempos de crisis. Esta carencia de legitimidad explica esta apelación a una figura jerárquica del Estado provincial respecto al Consejo, y no se resumía solo en el pedido del comisario, sino que un grupo de vecinos también dirigió su telegrama al ministro de Gobierno, donde alertaban por la salubridad de la población y reclamaban la autorización de Martínez Olano en dicho contexto:

43 Idem.

44 AGPSF, MG, tomo 30, 1895, exp. 12. 
En motivo del flagelo que nos amenaza por el contacto en que estamos con Rosas y esa capital y habiendose retirado para Cordoba el Sr. Carlos Achaval pedimos a SS. ponga en conosimiento del Sr. gobernador que encuentrase aquí el Sr. Martinez Olano, medico extranjero que esta inhibido por el Consejo de Higiene a ejercer su profesión. Por las circunstancias espuestas pedimos a SS. y al Sr. Gobernador la autorización [...] para que este Sr. pueda ejercer la medicina y poder a la vez tomar medidas preventivas contra la epidemia reinante. Esperamos se nos atienda urgente. Saludamos al Sr. Ministro. Adolfo K. Gielz, I. Maistany, Antonio I Rodriguez, Choistensen y Cia, Koennig y Cia, Angel Bagliente, I del Casto. ${ }^{45}$

Seis días después, el Ministerio de Gobierno pasa la nota al Consejo para su evaluación, que se expide en pocos días negando la posibilidad de autorizar a Martínez Olano y enfatizando su carácter «ilegal» no solo desde su práctica, sino también por no haber abonado las multas impuestas. La lectura sesgada que realizaban respecto a la normativa y a las posibilidades mismas de reunir las condiciones para llevarlas a cabo es evidente:

Los artículos del Reglamento prohiben terminantemente se faculte al ejercicio de la medicina a personas que carezcan de títulos universitarios. Por esta causa se prohibió al Sr. Martinez Olano ejerciera el arte de curar hasta tanto no presentara documentos que comprobasen su competencia. No lo hizo, persistiendo por varias ocasiones, por lo que se le aplicaron dos multas; una de $\$ 100$.-, satisfecha, y la otra de $\$ 200$.- impaga aún, y cuya efectividad se ha recomendado al Gefe Político de Rafaela, por no haberlo hecho el Juez de Paz de Sunchalez. El Consejo no podria permitir se facultara al Sr. Martinez Olano, como o solicitan, por las razones expuestas, y porque será abandonar la suerte de aquellos habitantes en manos de un particular, lo que significa acarrearse la responsabilidad de los actos de aquel. Por otra parte, si el mal llegara a amenazar a Sunchalez, se aconsejaría inmediatamente el envío de un facultativo. Por hoy, esta Corporación no tiene a quien indicar se le proponga la plaza dejada por el Dr. Achaval. Candido Pujato. ${ }^{46}$

Sucede entonces que tras esta petición de la comunidad por la habilitación de Martínez Olano y la reiterada negativa de Pujato, el 7 de febrero de 1895 el jefe político de Rafaela respondió al recurso administrativo presentado por el presidente del Consejo el 20 de diciembre del año anterior, donde informaba que no fue posible darle curso, dado que «el Sr. Martinez Olano se ha aucentado de "sunchales", ignorando su domicilio». ${ }^{47}$ Esta respuesta no logró otro resultado que exacerbar aun más al presidente del

45 Idem.

46 Idem.

47 «Presidente del Consejo de Higiene acompaña notas quejándose de la demora de algunas autoridades para los asuntos de dicho Consejo», AGPSF, MG, tomo 30, 1895, exp. 30. 
Consejo, quien se dirigió con el asunto al ministro de Gobierno, apuntando contra el accionar del funcionario de Rafaela, a quien acusaba de obrar deliberadamente en contra de las disposiciones requeridas, y en favor del curandero:

Para que su SS pueda darse cuenta del retardo notable con que proceden las autoridades de campaña en asuntos de suma importancia, que este Consejo recomienda, me permito acompañar la nota $\mathrm{n}^{\circ} 890$ en la que se solicitaba averiguaran la veracidad de lo expresado en la nota 877. Sírvase fijarse SS en la fecha de la diligencia, en la forma en que se hace, en el poco interés que se demuestra, y tendrá forzosamente, que convenir que la buena voluntad de esta Corporación se verá siempre quebrantada por la decidia, cuando no por la mala intención de empleados sin escrúpulos. ${ }^{48}$

Esta última requisitoria no fue bien recibida por el jefe político de Rafaela, que en su respuesta agrega un dato hasta entonces ausente: él era nuevo en el cargo, habiendo asumido a solo veinte días de presentado el recurso de Pujato. Con lo cual, en términos administrativos, había dado cumplimiento a los procedimientos correspondientes.

\begin{abstract}
Al hacerme cargo de esta jefatura, el 14 de enero ppdo. Dediqué los primeros días a examinar los expedientes atrasados entre los que se hallaba el presente del Consejo de Higiene que se remitió a esta Jefatura el 20 de diciembre último; procuré indagar cuál fuera el paradero del Sr. Martinez Olano y como supe que no se hallaba en Sunchales ni cual era su residencia se lo hice notar en el citado expediente al Señor Presidente con fecha 7 del actual pues nada podía hacer. Por tanto creo que no es pertinente la falta que en la nota 14 del actual me atribuye el Señor Presidente del referido Consejo, puesto que desde el 20 de diciembre fecha de la nota hasta últimos de enero en que yo me enteré del asunto no es culpa mía que no se tramitara; y la prueva de mi celo en pro de este asunto es palpable, pues a los 7 días ya lo devolví informado diciendo que ignoraba el paradero del Sr. Martinez y no hallándose en mi Departamento mal podría dar cumplimiento a lo que se solicitaba. ${ }^{49}$
\end{abstract}

Con esta respuesta, es posible analizar el caso de manera inversa a lo que las fuentes plantean inicialmente. Si en un principio es evidente que los policías y funcionarios locales estaban protegiendo a Martínez Olano al afirmar que no era posible dar con su paradero, la intervención del nuevo jefe político muestra que la imposibilidad de llevar a cabo la voluntad del Consejo respondía, en verdad, a situaciones estrictamente contingentes. Es menester, empero, que retengamos la actitud de Pujato. A pesar de que hemos reconocido una particular predisposición en la actitud de este

48 Idem.

49 Idem. 
personaje al analizar el caso del espiritista Juan Quinteros, sus sospechas sobre las trabas que la comunidad y los agentes del Estado a nivel local podían estar interponiendo en su requisitoria contra Martínez Olano nos da el indicio de que ese escenario era más que posible y esperable. El hecho mismo de que los funcionarios encargados del ejercicio de la violencia legítima no llevasen a cabo las instrucciones del Consejo evidencia que, a pesar de su incremento de atribuciones y su voluntad reguladora, estos intentos no encontraban un correlato en el tejido social, después de casi treinta años de funcionamiento.

El caso que sigue también da cuenta de una nueva forma de canalización de los conflictos por parte del Consejo, y un recrudecimiento de los controles y de los mecanismos administrativos para lidiar con quienes ejercían el arte de curar sin autorización. En 1901 José Lardone, médico cirujano, se dirige al ministro de Gobierno para denunciar el accionar de un supuesto médico alemán, Teofilo Kuntz, que competiría con él en la prestación de servicios, ejerciendo la medicina sin estar autorizado por el Consejo. A diferencia de los casos que hemos revisado, aquí el médico «oficial» alega haber sido consultado en casos donde el galeno germano había intervenido y cobrado honorarios, que detalla con nombre y apellido en su nota:

\begin{abstract}
Hace un año más o menos se ha establecido en el pueblo de Armstrong de este Departamento, un señor cuyo apellido es Teofilo Kuntz, de nacionalidad alemana, el cual ejerce la medicina sin autorización del Consejo de Higiene. El que suscribe ha sido llamado varias veces para asistir enfermos en dicha localidad, los cuales habían ya sido visitados por dicho Sr., a quienes había recetado y cobrado sus honorarios. Este señor se presenta como médico y según referencia, ha practicado una autopsia. Como este señor ha sido apercibido por el anterior Consejo de Higiene, así reitera la denuncia para los proveimientos necesarios. Confiando en que SS. hará respetar los intereses y el decoro que merece el arte de curar..$^{50}$
\end{abstract}

El avance del procedimiento administrativo implicó al jefe político del departamento San Martín, quien fue compelido a comprobar la veracidad de la denuncia de acuerdo con las disposiciones vigentes, y por ello deriva el asunto al juez de paz de la localidad de Sastre para que inicie un proceso administrativo. Aquí veremos la novedad de este caso: se convoca a los sujetos mencionados en la denuncia de Lardone y se les toma declaración. El mismo día que dicho juez de paz recibe la solicitud, comienza el proceso

50 AGPSF, MG, tomo 356, 1901. 
y toma declaración a un testigo, agricultor e italiano, de cuarenta y cinco años, que dice conocer a Kuntz - a quien «corresponden las generales de la ley»- pero ignora la razón por la que se lo convoca. Cuando el juez pregunta si el curandero utilizó su casa para atender y recetar, el testigo responde que cuatro meses atrás su esposa enfermó y recurrió a Kuntz pidiéndole algunos medicamentos, y este cobró una pequeña suma de dinero por su consulta y prescripción. El interrogatorio cierra con una pregunta sobre si Kuntz continúa atendiendo personas en su vivienda y si conoce de otras curas que haya realizado, a lo cual el campesino responde que no ocupó nuevamente sus servicios y que desconoce de otras atenciones. Un segundo testigo fue Juan Gabatto, también agricultor e italiano, de cuarenta y seis años, e ignorante del motivo por el que se lo convoca. Si bien dice conocer a Kuntz, en este caso afirma que «no le corresponden las generales de la ley», y respecto a los servicios que le prestó, declara que ocho meses atrás su padre estuvo enfermo, ante lo cual el sanador recetó un medicamento en forma de inyecciones, desconociendo si las mismas provenían de una farmacia, y como pago le ofreció un regalo, dado que Kunz no puso precio a su trabajo. El tercer testigo convocado, Pedro Pumero, era considerablemente más joven — veintiún años—, y como los dos anteriores, agricultor e italiano, aunque soltero. Como el caso anterior, afirma que al curandero extranjero no le corresponden las generales de la ley, y que su contacto con él fue por una enfermedad de su madre, ante lo cual no recuerda si Kuntz prescribió un medicamento, pero sí que sugirió aplicar hielo en la cabeza. Por dichos servicios Kuntz percibió dos pesos moneda nacional, y al preguntar si conocía otras personas a las cuales Kutnz había prestado servicios, el testigo dice desconocer, pero había atendido a una hermana suya que falleció.

Dos de los sujetos mencionados en la requisitoria de Lardone no residían en dicho poblado, sino en la Colonia de Carlos Pellegrini, con lo cual se solicita y faculta al juez de paz de la misma para ordenar comparezcan ante él a prestar indagatoria. El primero en hacerlo es un agricultor llamado José Mondino, italiano de veintidós años, quien dice conocer a Kuntz por haber enfermado dos meses atrás, ante lo cual se lo convoca. El sanador hizo dos visitas y cobró por sus servicios dos pesos, desconociendo el testigo si realizó otras curaciones. La segunda testigo es Ángela Rinaudi de Perrotti, italiana de cincuenta y tres años, casada —único caso donde se aclara que lee y escribe- y con seis años de residencia en el país. Esta mujer dedicada a los «quehaceres domésticos» afirma que solicitó los servicios de Kuntz a comienzos del año, quien hizo una sola visita y cobró tres pesos. 
Una vez finalizadas las indagatorias requeridas, se remiten las doce fojas al Ministerio de Gobierno, donde se deriva el expediente a la Inspección de Policía, que resuelve que Kuntz ejerce la medicina y cobra honorarios, ante lo cual resta saber si estaba autorizado por el Consejo de Higiene Nacional o bien por el de Rosario o Santa Fe, para determinar finalmente si era preciso o no aplicar penas. Tras dos meses, el Ministerio de Gobierno cierra el caso el veinticuatro de agosto alegando que se desestima la solicitud que cuestionaban las actuaciones de Teófilo Kuntz una vez que este solicitó autorización para ejercer su profesión, la cual aparentemente fue aprobada. Este caso nos muestra el cambio en las prácticas de control del Consejo, que comenzaba a articular sus procedimientos con otras instancias burocráticas del Estado y, con ello, a limitar una de las aristas que permitían a los sanadores populares continuar con sus actividades. Si bien podemos ver en los testigos que continuarían recurriendo a los servicios del curandero, los cuadros estatales comenzaron a desarrollar procesos administrativos reglados, a partir de los cuales la aplicación de las multas y las prohibiciones sobre estos sujetos se tornaba cada vez más severa.

En ese mismo año, Walter Sevilla, médico de policía en San Cristóbal se dirige al ministro de Gobierno en «su lucha contra la audacia de los curanderos» para solicitar que no se autorice a Francisco Beltramino a ejercer la profesión, por no haber defendido su tesis, o bien se lo reubique para que no perjudique a los diplomados. ${ }^{51} \mathrm{Su}$ denuncia incluía, además, un pedido para que sus recetas no se despachen en las farmacias locales, lo cual demuestra que el problema no se circunscribía solamente al accionar del sanador, sino también a la connivencia de sus prácticas con los otros actores locales que participaban de las prestaciones de salud. Si bien cuando se redireccionó su petición al Consejo este reconoció la justicia de su reclamo, al mismo tiempo habilitó a Beltramino para ejercer en alguna zona donde no intervenga ni compita con los «oficiales». Este dato es significativo, en tanto reconoce una excepción a la prohibición que regía sobre los médicos extranjeros y los idóneos desde la reforma del Consejo en 1887, siempre que se resguardase la prioridad del diplomado oficial e inscripto, despejando así su posible competencia.

$\mathrm{Al}$ año siguiente, se registraron dos denuncias contra practicantes ilegales de la medicina que ponen en evidencia las tensiones surgidas no solo entre practicantes diplomados y populares, sino también entre los prime-

51 AGPSF, MG, tomo 358, 1901, exp. 7. 
ros y las instituciones que debían velar por ellos, sea el Consejo, sean las autoridades locales. La primera de ellas se originó en Sunchales, donde ya observamos casos similares, y corresponde al doctor Miguel Alarcón, quien instruye «diligencias sumarias» contra Miguel Piccone por ejercer el arte de curar sin autorización. Uno de los aspectos relevantes del caso reside en que el motor de su denuncia no apuntaba a censurar su ejercicio. Piccone, de nacionalidad italiana pero sin reválida oficial del Consejo de Higiene local, aparentemente difamaba a Alarcón e incumplía las notificaciones recibidas en este sentido por contar, de acuerdo al denunciante, con la «protección» de las autoridades locales. ${ }^{52}$

El segundo caso, acaecido a fines de mayo de ese año, fue recogido por el juez de paz de la localidad de San Genaro, donde el médico local denunció a Francisco Aresse, un sujeto que ejercía el arte de curar de manera ilegal, disputando su clientela, y con una considerable legitimidad social:

Desde hace cinco meses a esta parte que el médico de la localidad Dn Nicolas de Feo, viene quejándose con insistencia contra un curandero llamado Francisco Aresse, que usurpándole su arte ejerce también la medicina clandestinamente, en perjuicio no solo del médico de Feo con título de facultad extranjera revalidado en el país, si que también haciendo víctimas a los humildes o ignorantes habitantes de este pueblo, a quienes induce a creer que la aplicación de su arte tiene la virtud de la «panacea»..$^{33}$

El problema de fondo en este caso fue que el Consejo mismo no daba respuesta a los requerimientos del profesional diplomado, por encontrarse en recambio de autoridades, y por ende temporalmente acéfalo. Esto motiva su apelación a otra instancia burocrática del Estado para canalizar su demanda de reconocimiento y limitar la práctica de Aresse. En su petición, además de bregar por el reconocimiento de su experticia en comparación al curandero, apelaba al bienestar de la población y a los «abusos» que el accionar de este tipo de sujetos representaba sobre la población local:

Como se trataba de funciones que incumbían exclusivamente atender al Consejo de Higiene de esa capital por repetidas veces se insinuó al médico de Feo que recurriera a dicha corporación, resultando que por la acefalía en que ella se encontraba esos reclamos no le han sido atendidos. Ahora bien, como la libertad con que dicho curandero ejerce el arte de curar raya en el abuso en perjuicio de intereses no ya particulares del médico de Feo sino en lo que son más caros, en los intereses de vidas de los vecinos

52 AGPSF, MG, tomo 375, 1902, exp. 16.

53 AGPSF, MG, tomo 376, 1902, exp. 46. 
que aún creen en la eficacia del arte de estos adivinos; y es por ello que llevo este hecho a conocimiento de SS. el Sr. Ministro, pidendole quiera dignarse ilustrarme en el proceder que debe adoptarse para reprimir el abuso denunciado. ${ }^{54}$

El caso fue trasmitido del Ministerio de Gobierno al Consejo, el cual informó que no poseía constancia de haber prohibido a Aresse ejercer su oficio, pero que en todo caso, la notificación del juez de paz sobre su reincidencia será suficiente para aplicar la multa correspondiente. Para ello, además, el órgano corporativo habilitó al magistrado para notificar al sanador que de continuar con su práctica se implementaría el artículo trece del reglamento, por el cual se lo multaría de manera creciente de acuerdo al número de reincidencias. Estos procedimientos significaron, aparentemente, el final del caso, y entendemos que evidencian una nueva lógica respecto a los observados previamente. Si bien el Consejo no fue la entidad que respondió originalmente al reclamo de uno de sus miembros corporativos, fue la autoridad local —representada en el juez de pazquien tomó a su cargo la defensa del médico que proclamaba vulnerados sus derechos. Esto representa una clara diferencia con lo acontecido durante el último tercio del siglo, donde los galenos avanzaban en soledad contra sus «adversarios» en la prestación de servicios de salud, y respondía, en primer lugar, a que los incipientes cuadros estatales no se habían autonomizado en el ejercicio de su función, y luego fueron - lentamente- incorporando nuevas atribuciones. Pero también, en segundo término, podemos agregar que, en su profesionalización, estos cargos de autoridad civil comenzaban a ser desempeñados por personajes letrados que podían empatizar más fácilmente con un profesional diplomado del arte de curar frente a otro «ilegal».

\section{Reflexiones finales}

En este trabajo hemos procurado aportar a dos problemas que atañen al campo de la historia social de la salud y la enfermedad. Por una parte, a los «avatares» del proceso de medicalización social en una provincia de $\mathrm{Ar}$ gentina; por otra, a las derivas de la profesionalización y legitimación médica a partir de una serie de casos que ponen en tensión la supuesta linealidad

54 Idem. 
que las historias tradicionales de la medicina han atribuido al accionar de los médicos y su recepción «positiva» por parte de la sociedad. En efecto, si algo evidencian las fuentes analizadas es, por una parte, la escasa legitimidad con que contaban los galenos y su saber académico, mucho más patente en sectores «rurales» o ajenos a la vida citadina, donde no se encuentran denuncias documentadas. Ahora bien, por otra parte, tanto las solicitudes de los vecinos como las presentaciones de los diplomados dan cuenta de la sinuosidad y conflictividad que signó la profesionalización de la medicina en términos empíricos. Es importante reconsiderar en este proceso la esfera de la «estatalidad», en especial a partir de su no acompañamiento inicial al reclamo de los galenos. Si bien las agencias y el funcionariado estatal se caracterizaron durante el siglo XIX y principios del XX por su condición incipiente, frágil y de fronteras lábiles entre los intereses privados y públicos, los casos abordados muestran cómo, a pesar de inscribir sus reclamos y su agenda en la estructura estatal provincial, esto no aseguraba a los médicos el cumplimiento de sus disposiciones en el entramado territorial, donde su legitimidad era más bien escasa.

Cobra una dimensión especialmente significativa en este artículo el abordaje de estos procesos a la luz de denuncias canalizadas a través de distintas instancias del Estado en varios sentidos. Por un lado, por el trabajo de reunión y sistematización de las mismas en el marco de un conjunto más extenso de fuentes referidas al Consejo de Higiene, pero también y más importante aun, por la localización y rescate de los conflictos entre diplomados y sanadores populares con anterioridad a la existencia de tal institución.

Por otro lado, estas fuentes en su conjunto ponen de manifiesto un aspecto central y previo a cualquier análisis, esto es, la emergencia misma del conflicto entre prácticas de curar, y la progresiva delimitación de un campo de polaridad donde un saber y una terapéutica específicos se erigían como legítimos frente a otro que era preciso desacreditar. En dicha tarea, se apelaba a un tímido criterio de cientificidad frente a la ignorancia de los curanderos, donde vemos configurarse un paradigma propio de la modernidad occidental donde el saber científico se propone como única instancia de saber autorizada, y con capacidad de anulación de cualquier otra. Frente a la constatación inicial de los médicos de que la apelación a la «charlatanería» no resultaba efectiva en su cruzada, la manera más usual de desacreditar a sus oponentes fue, por un lado, insistir en el riesgo al que sometían a la población que a ellos recurría. Pero también, por otro, un aspecto presente en 
la casi totalidad de las denuncias pasa por la difamación de los diplomados; y no es preciso insistir en el hecho de que, en estas sociedades históricas, la apelación al honor y a su respeto era un argumento más audible para las autoridades.

De todo esto rescatamos que, por encima de los intentos de los diplomados, no solo es la legitimidad de su figura como expertos de la salud lo que las fuentes nos informan, sino también la de los saberes que los erigían como tales. Con ello, es posible apreciar la historicidad de la medicina como conjunto de saberes válidos e incuestionables en la promoción de procesos de salud, así como de la medicalización social como proceso unilateral y totalizante.

En un primer momento, entonces, observamos la dinámica de las denuncias de los diplomados y las solicitudes «populares» ante epidemias en un contexto previo a la reglamentación e institucionalización - formalde la corporación bajo la figura del Consejo de Higiene. Los casos contemplados hasta 1868 nos permiten ver los escasos recursos de los diplomados en términos de su posición en el Estado, sea por su lugar casi inexistente — recordemos que Teófilo Romang era médico del municipio de Esperanza pero no cobró su sueldo por años-, o bien por la ausencia de un espacio de defensa corporativa en su interior. Asimismo, las solicitudes de los vecinos muestran no solo la deficiente estructura con que el incipientemente estabilizado Estado provincial contaba ante los brotes epidémicos, sino también la presencia misma de figuras arraigadas en la comunidad que desarrollaban el arte de curar previo a la intervención de estatal.

En un segundo momento del análisis, observamos que las quejas contra los practicantes ilegales comenzaban a ser abordadas directamente por el Consejo de Higiene - a partir de la mediación del Ministerio de Gobierno-, involucrando, además, a los farmacéuticos como miembros de una esfera común regulada por el órgano, el cual dejaba ver su voluntad de reconocimiento como instancia de contralor en lo referido a las temáticas de su incumbencia. Ahora bien, este reconocimiento de los intereses particulares de los galenos como interés común del conjunto por parte del Estado no dejaba de ser estrictamente formal. Los casos que analizamos en la segunda sección del artículo dan cuenta de la inexistente articulación entre dichos intereses y la dinámica de cada poblado.

En la denuncia al curandero Martínez Olano de 1894, emergen con claridad estas tensiones: por una parte, la (escasa) legitimidad local de los médicos en el pueblo donde se desata la misiva contra el sanador. En 
segundo lugar, la «flexibilidad» de las estructuras administrativas y represivas estatales a la hora de llevar a cabo la multa dispuesta por el Consejo, lo cual evidencia algún tipo de connivencia o al menos aprobación de la comunidad de su tarea. Por último, las encendidas respuestas del presidente del Consejo expresan un grado de tensión considerable respecto al incumplimiento de la normativa referida al resguardo de la corporación, que llevaba casi tres décadas de funcionamiento.

Los casos abordados en la segunda sección del artículo muestran particularidades del proceso en otra clave. Por un lado, que las denuncias efectuadas por los médicos apuntaban a sujetos que no eran curanderos sino más bien médicos no autorizados por algún motivo ante el Consejo provincial, y aquí se hace presente un aspecto que atraviesa a todos los expedientes trabajados. No es solo una cuestión de legitimidad lo que preocupa a los diplomados, sino también de competencia en la práctica. Mientras que en un primer momento las denuncias se focalizan en el ejercicio del arte de curar por sujetos «no capacitados» aunque con mayor legitimidad, con el paso de las décadas el foco se desplaza a aquellos médicos que amenazaban el monopolio local de aquellos sujetos que habían logrado la autorización estatal. Con ello, el visto bueno de la corporación institucionalizada pasó a jugar un rol de árbitro de la competencia interna al interior de la medicina oficial.

Por otra parte, el final del siglo XIX evidencia cambios significativos en los procedimientos administrativos del Estado - con el desarrollo de las indagatorias y su uso como prueba-, así como una mayor «predisposición» de los funcionarios de turno para ceñirse a los mismos. No es menor considerar que el componente letrado al interior de la burocracia estatal debía ser más considerable, y con ello su cercanía y «creencia» en la medicina en tanto saber académico legitimado institucionalmente. Entendemos que ello aun no implica un reconocimiento pleno por parte del Estado, sino más bien un cambio en la composición de sus elites y su funcionariado, que podía facilitar el devenir de los pedidos de la corporación.

Es claro que el número de denuncias y solicitudes trabajadas aquí no permite arrojar conclusiones definitivas sobre procesos tan complejos y con múltiples aristas, como fueron la medicalización social, la profesionalización de la medicina y, en particular, la legitimidad del médico como agente primordial y hegemónico de la salud. Entendemos, sin embargo, que en su variedad y extensión temporal permiten vislumbrar la conflictividad y, sobre todo, los denodados esfuerzos que conllevó a la comunidad médica 
diplomada su reconocimiento como un actor de referencia en la búsqueda del restablecimiento de un estado de «salud», un camino en el cual la extensión del saber médico sobre la regulación de la vida de las poblaciones no necesariamente fue exitosa, sino más bien conflictiva.

Recibido el 23 de enero de 2017 Segunda versión el 4 de agosto de 2017 Aceptado el 5 de septiembre de 2017

\section{Referencias bibliográficas}

Amodio, Emanuele, «Curanderos y médicos ilustrados. La creación del Protomedicato en Venezuela a finales del siglo XVIII», Asclepio. Revista de Historia de la Medicina y de la Ciencia, XLIX, Madrid, 1997, 95-29.

Armus, Diego, «La enfermedad en la historiografía de América Latina moderna», Asclepio. Revista de Historia de la Medicina y de la Ciencia, 54, 2, Madrid, 2002, 41-60.

Armus, Diego, La ciudad Impura, Editorial Edhasa, Buenos Aires, 2007.

Armus, Diego, «Medicina casera, remedios y curanderos en los inicios de la medicalización de la ciudad moderna. Buenos Aires, 1870-1940», Tempos Históricos, 20, Paraná, 2016, 47-80.

Bohoslavsky, Ernesto y Di Liscia, María Silvia, «La profilaxis del viento. Instituciones represivas y sanitarias en la Patagonia argentina, 1880-1940», Asclepio. Revista de Historia de la Medicina y de la Ciencia, LX, 2, Madrid, 2008, 187-206.

Bohoslavsky, Ernesto y Godoy Orellana, Milton (eds.), Construcción estatal, orden oligárquico y respuestas sociales. Argentina y Chile 1840-1930, Buenos Aires, UNGS, Universidad Academia de Humanismo Cristiano, Prometeo Libros, 2010.

Bohoslavsky, Ernesto y Soprano, Germán (eds.), Un Estado con rostro humano. Funcionarios e instituciones estatales en Argentina (desde 1880 a la actualidad), Buenos Aires, Universidad Nacional de General Sarmiento, Prometeo Libros, 2010.

Bonaudo, Marta (dir.), Liberalismo, Estado y orden burgués (1852-1880), Tomo IV de la Colección Nueva Historia Argentina, Buenos Aires, Sudamericana, 2007 [1999].

Bubello, Juan Pablo, Historia del esoterismo en la Argentina. Prácticas, representaciones y persecuciones de curanderos, espiritistas, astrólogos y otros esoteristas, Buenos Aires, Biblos, 2010.

Carbonetti, Adrián, «La Construcción del poder médico en el marco de la formación del Estado Nacional y la reestructuración de los Estados Provinciales. 
El caso de Córdoba. 1869-1914», Terceras Jornadas de Historia de Córdoba, Córdoba, Junta Provincial de Historia, 1997, 241-257.

Carbonetti, Adrián; Aizenberg, Lilia y Rodríguez, María Laura, «La Historia Social de la Salud y la Enfermedad: conformación, logros y desafíos», Revista Estudios, 30, Córdoba, 2014, 145-157.

Castel, Robert, La metamorfosis de la cuestión social. Una crónica del salariado, Buenos Aires, Paidós, 1997 [1977].

Dahhur, Astrid, «Ataques a curanderos. La medicina y su lucha por el control social de la población en los primeros años del siglo XX», XIV Jornadas Interescuelas/Departamentos de Historia, Universidad Nacional de Cuyo, Mendoza, 2013, https://www.aacademica.org/000-010/530.

Di Liscia, María Silvia, Saberes, Terapias y Prácticas Médicas en Argentina (17501910), Madrid, Consejo Superior de Investigaciones Científicas, 2003.

Di Liscia, María Silvia, «Reflexiones sobre la nueva historia social de la salud y la enfermedad en Argentina», en Carbonetti, Adrián y González Leandri, Ricardo (eds.), Historias de salud y enfermedad en América Latina, Siglos XIX y XX, Córdoba, Editorial CEA, CONICET, 2008, 15-47.

Faberman, Judith, Las Salamancas de Lorenza. Magia, Hechicería y curanderismo en el Tucumán colonial, Buenos Aires, Siglo XXI, 2005.

Fernández, Sandra R. (comp.), Más allá del territorio. La historia regional y local como problema. Discusiones, balances y proyecciones, Rosario, Prohistoria, 2007.

Fernández, Sandra R., «El revés de la trama: contexto y problemas de la historia regional y local», en Bandieri, Susana; Blanco, Graciela y Blanco, Mónica (coords.), Las escalas de la historia comparada. Tomo 2: Empresas y empresarios. La cuestión regional, Buenos Aires, Miño y Dávila, 2008, 233-246.

Fernández, Sandra y Dalla Corte, Gabriela, Lugares para la historia: espacio, historia regional e historia local en los estudios contemporáneos, Rosario, UNR Editora, 2001.

Ferreira, Luiz Otávio, «Medicina impopular. Ciencia médica e medicina popular nas páginas dos periódicos científicos (1830-1840)», en Chaloub, Sydney et al. (orgs.), Artes e ofícios de curar no Brasil. Capítulos de História Social, Campinas, Editora Unicamp, 2003, 101-122.

Fleitas, Mirta, «iQueremos a Mano Santa!: actores y significados de una revuelta popular acontecida en 1929 en San Salvador de Jujuy», Salud Colectiva, vol. 3, 3, Remedios de Escalada, 2007, 301-313.

Gallo, Ezequiel, La pampa gringa. La colonización agrícola en Santa Fe (18701895), Buenos Aires, Edhasa, 2004 [1983].

Garcilazo, Romina, «Recurrir a las autoridades por una justa causa. El derecho de petición en la ciudad de Rosario (Argentina) 1883, 1884-1890», Temas Americanistas, 33, Sevilla, 2014, 130-152.

González Leandri, Ricardo, Curar, persuadir, gobernar. La construcción histórica de la profesión médica en Buenos Aires, 1852-1886, Madrid, CSIC, 1999. 
González Leandri, Ricardo, «El Consejo Nacional de Higiene y la consolidación de una elite profesional al servicio del Estado. Argentina, 1880-1900», Anuario de Estudios Americanos, 61,2, Sevilla, 2004, 571-593.

González Leandri, Ricardo, «La consolidación de una inteligentzia médico profesional en Argentina: 1880-1900», Diálogos. Revista Electrónica de Historia, 7, 1, Costa Rica, 2006, 36-78.

González Leandri, Ricardo, «Itinerarios de la profesión médica y sus saberes de Estado. Buenos Aires, 1850-1910», en Plotkin, Mariano y Zimmermann, Eduardo (comps.), Los saberes del Estado, Buenos Aires, Edhasa, 2012, 125-152.

Hourcade, Eduardo y Godoy, Cristina, «La economía santafesina en la segunda mitad del siglo XIX», en Ascolani, Adrián (comp.), Historia del sur santafesino. La sociedad transformada (1850-1930), Rosario, Ediciones Platino, 1993, 19-40.

Molina Jiménez, Iván y Palmer, Steven, La voluntad radiante. Cultura impresa, magia y medicina en Costa Rica (1897-1932), San José, Editorial Porvenir y Plumsock Mesoamerican Studies, 1996.

Palmer, Steven, «La "voluntad radiante" del Profesor Carbell. Medicina popular y populismo médico en Costa Rica en el decenio de 1930», en Armus, Diego (ed.), Entre médicos y curanderos. Cultura, historia y enfermedad en la América latina moderna, Buenos Aires, Grupo Editorial Norma, 2002, 259-292.

Pascual, Cecilia M., «La epidemia de cólera como condensador de sentidos. Culturas urbanas, narraciones clínicas y políticas higiénicas en Rosario, Argentina, 1886-1887», História Ciências, Saúde-Manguinhos, 24, 2, Río de Janeiro, 2017, 295-311.

Plotkin, Mariano y Zimmermann, Eduardo (comps.), Los saberes del Estado, Buenos Aires, Edhasa, 2012.

Podgorny, Irina, Charlatanes. Crónicas de remedios incurables, Buenos Aires, Eterna Cadencia, 2012.

Podgorny, Irina, Charlatanería y cultura científica en el siglo XIX, Madrid, Libros de la Catarata, 2015.

Prieto Agustina, «Rosario. Epidemias, Higiene e higienistas en la segunda mitad del siglo XIX», en Lobato, Mirta (ed.), Política, médicos y enfermedades. Lecturas de la historia de la salud en la Argentina, Mar del Plata, Biblos, 1996, 57-71.

Reguera, Andrea (coord.), Los rostros de la modernidad. Vías de transición al capitalismo. Europa y América siglos XIX y XX, Rosario, Prohistoria, 2009.

Revel, Jacques, Un momento historiográfico. Trece ensayos de historia social, Buenos Aires, Manantial, 2005.

Rivero, María Dolores; Carbonetti, Adrián y Rodríguez, María Laura, «Alternativas al saber diplomado en la escena pública: una aproximación al curanderismo a partir de la prensa escrita de las ciudades de Córdoba y de Buenos Aires, Argentina en la década de 1920», Historia y Sociedad, 33, Medellín, 2017, 19-43. 
Rodríguez, María Laura; Carbonetti, Adrián y Andreatta, María Marta, «Prácticas empíricas y medicina académica en Argentina. Aproximaciones para un análisis cuanticualitativo del Primer Censo Nacional (1869)», Historia Crítica, 49, Bogotá, 2013, 81-108.

Sedrán, Paula; Carbonetti, Adrián y Allevi, José Ignacio, «Juan P. Quinteros, espiritista. Disputas por los sentidos legítimos del arte de curar. Santa Fe, fines del siglo XIX», Revista de Indias, Madrid, en prensa.

Sowell, David, «Andanzas de un curandero en Colombia: Miguel Perdomo Neira y "la lucha entre el buen sentido y la ignorancia ciega"», en Armus, Diego (ed.), Entre médicos y curanderos, Buenos Aires, Grupo Editorial Norma, 2002, 71-103.

Suriano, Juan (comp.), La cuestión social en Argentina. 1870-1943, Buenos Aires, Ed. La Colmena, 2000. 